\title{
Kindlin Assists Talin to Promote Integrin Activation
}

\author{
Z. Haydari ${ }^{1}$, H. Shams ${ }^{1}$, Z. Jahed ${ }^{1}$, M.R.K. Mofrad ${ }^{*}$
}

${ }^{1}$ These authors contributed equally to this paper

*Corresponding Author 


\begin{abstract}
Integrin $\alpha \operatorname{IIb} \beta 3$ is a predominant type of integrin abundantly expressed on the surface of platelets and its activation regulates the process of thrombosis. Talin and kindlin are cytoplasmic proteins that bind to integrin and modulate its affinity for extracellular ligands. While the molecular details of talin-mediated integrin activation are known, the mechanism of kindlin involvement in this process remains elusive. Here, we demonstrate that the interplay between talin and kindlin promotes integrin activation. Our all-atomic molecular dynamics simulations on complete transmembrane and cytoplasmic domains of integrin $\alpha \mathrm{IIb} \beta 3$, talin1 F2/F3 subdomains, and kindlin2 FERM domain in an explicit lipid-water environment over microsecond timescale, unraveled the role of kindlin as an enhancer of the talin interaction with the membrane proximal region of $\beta$-integrin. The cooperation of kindlin with talin results in a complete disruption of salt bridges between R995 on $\alpha$ IIb and D723/E726 on $\beta 3$. Furthermore, kindlin modifies the molecular mechanisms of inside-out activation by decreasing the crossing angle between transmembrane helices of integrin $\alpha I I b-\beta 3$, which eventually results in parallelization of integrin dimer. In addition, our control simulation featuring integrin in complex with kindlin reveals that kindlin binding is not sufficient for unclasping the inner membrane and outer membrane interactions of integrin dimer, thus ruling out the possibility of solitary action of kindlin in integrin activation.
\end{abstract}

\title{
Statement of Significance
}

Using the newly solved crystal structure of kindlin, we investigated, for the first time, the molecular mechanism of kindlin-mediated integrin activation through simultaneous binding of talin and kindlin. We demonstrate in atomist details how kindlin cooperates with talin to promote the activation of integrin $\alpha I I b-\beta 3$. 


\section{Introduction:}

Integrin plays a central role in regulating cell-matrix and cell-cell adhesion and is crucial for various signaling pathways involved in cell migration, proliferation, and differentiation $(1,2)$. Integrins are heterodimeric proteins composed of $\alpha$ and $\beta$ subunits that associate noncovalently. Each subunit consists of a large extracellular ectodomain (ECD), a single pass transmembrane helix (TM), and a short cytoplasmic tail (CT) (Fig. 1A) $(3,4)$. There are 24 different combinations of integrin $\alpha$ and $\beta$ subunits. Integrin $\alpha \operatorname{IIb} \beta 3$ is one of the well-known types that is only expressed in platelets and is necessary for the hemostatic function of platelets $(5,6)$. Integrins mediate bidirectional, inside-out and outside-in signaling across the membrane. Insideout signaling involves the interaction of intracellular proteins with the integrin CT, which modulates integrin's affinity for extracellular ligands $(7,8)$.

Three distinct conformational states of integrin have been determined: bent-closed or the resting state, extended-closed, and extended-open (Fig. 1A) (6). Integrin activation entails a conformational transition of integrin from a low-affinity (bent-closed) to a high affinity state (extended-open) (Fig. 1A) $(9,10)$. It was previously believed that talin was both necessary and sufficient for inducing integrin activation $(11,12)$, whereas recent studies contradict this notion (13-15) and suggest that kindlin is also needed as a co-activator of integrin $(13,16,17)$. For example, Moser et al. showed that kindlin3 knockout cells results in inactivated integrins despite presence of talin (18). Moreover, kindlin-associated diseases like Leukocyte Adhesion Disease III (LADIII), reflecting deficiencies of integrin function, show the necessity of kindlin in integrin activation (5).

Both talin (talin-1 and -2) and kindlin (kindlin-1, -2, and -3) isoforms include a FERM (4.1 protein, ezrin, radixin, moesin) domain, which is composed of four structural subdomains F0-F3 (19). Kindlin also features a pleckstrin homology $(\mathrm{PH})$ domain inserted into its $\mathrm{F} 2$ subdomain, which has been shown to directly interact with the lipid membrane (Fig. 1B) (20). Talin and kindlin both bind to the CT domain of integrin through their F3 subdomains. Specifically, talin binds to the NxxY motif at the membrane proximal region, while kindlin associates with this motif at the membrane distal part of CT suggesting that kindlin and talin can simultaneously bind to integrin tail and trigger activation (21). The molecular mechanisms of the interplay among talin and kindlin for inducing integrin activation are not yet well understood.

Recent experimental and computational studies have revealed important molecular details of integrin inside-out activation. These studies have suggested that the inner membrane clasp (IMC) between the CT domains of integrin subunits is critical for maintaining the closed state of integrin and unclasping induced by talin or other activators triggers activation (22-24), and that 
opening of IMC results in an increase in the crossing angle between $\alpha$ and $\beta$ monomers (25-27). However, all computational studies on integrin-mediated inside-out signaling to date have overlooked the role of kindlin due largely to lack of sufficient structural information $(7,28-30)$. In the present study we use the recently solved crystal structure of kindlin (31) to investigate the molecular mechanism of integrin activation through simultaneous binding of talin and kindlin. We use all-atomic microsecond-scale molecular dynamic simulations of $\alpha$ llb $\beta 3 \mathrm{TM} / \mathrm{CT}$ structure in an explicit lipid-water environment under the following three conditions: in complex with talin1 F2-F3 subdomains (IT), with kindlin2 (IK), and with both (ITK) to uncover the role of kindlin in integrin activation (Fig. 1C). Specifically, we explore how talin and kindlin binding affect allosteric transition of forces across the integrin molecule, i.e. from CT to TM, and eventually to the ectodomain. Our results show that kindlin 2 cooperates with talin 1 to facilitate integrin $\alpha$ llb $\beta 3$ activation by enhancing talin 1 interaction with the membrane proximal region of $\beta 3$-integrin. 

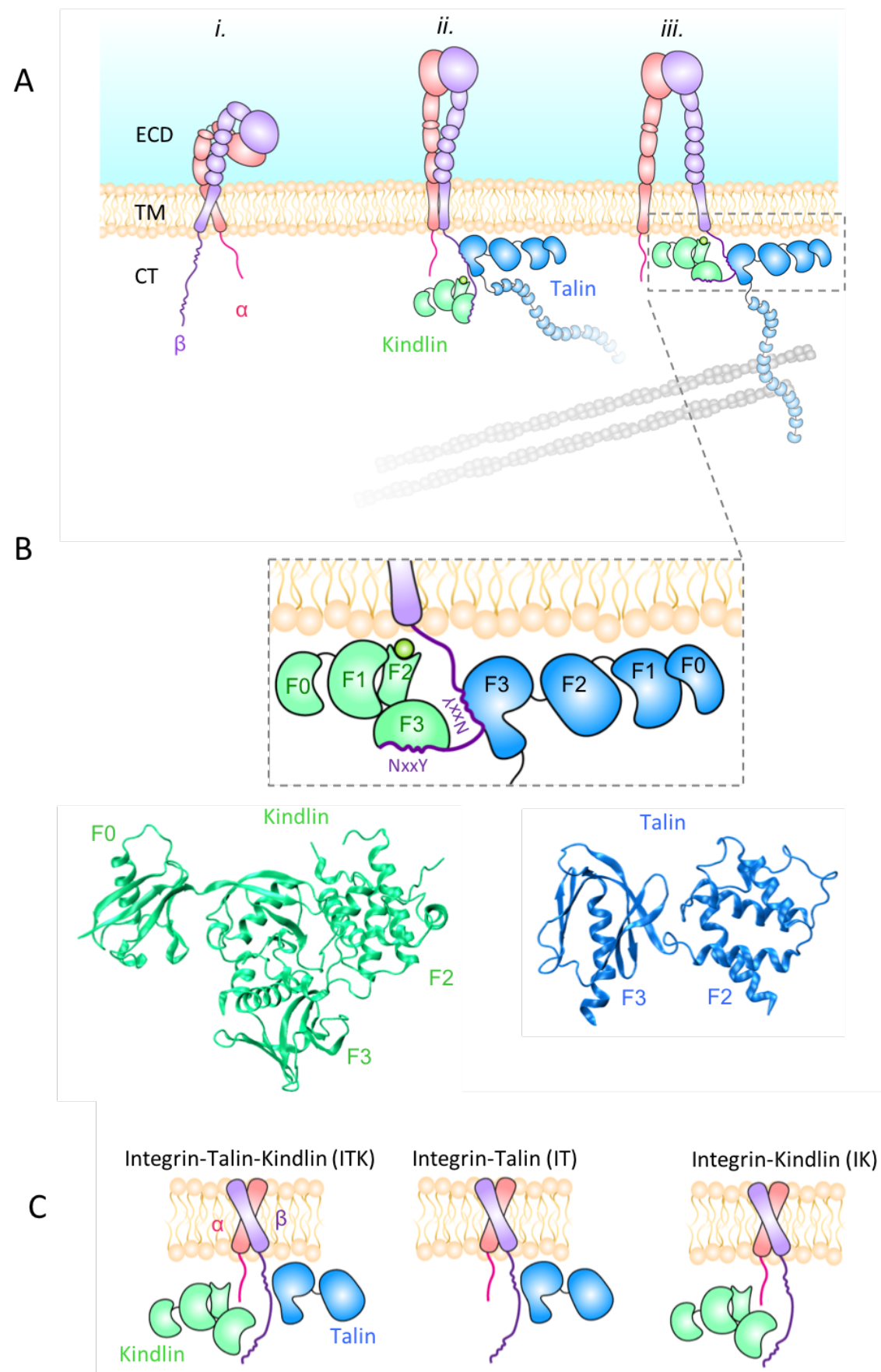

Figure1: Cytoplasmic interaction with the integrin tail mediate inside-out signaling

(A) Schematic representations of the three conformational states of integrin: i) bent-closed (inactive) ii) extendedclosed iii) extended-open (activated). Talin and kindlin are both involved in integrin activation by binding to the cytoplasmic domain of $\beta$. Talin association to the actin cytoskeleton provides a linkage between the extracellular environment and the cytoskeleton. (B) The F3 subdomain of talin and kindlin bind to the membrane proximal and membrane distal regions of $\beta$-integrin, respectively. (top). The crystal structure of kindlin2 (F0-F3 subdomains) and talin (F2-F3 domains). (C) Schematic representations of the three molecular models used in our simulations: integrin in complex with talin and kindlin (ITK), integrin in complex with talin (IT), and integrin in complex with kindlin (IK). 


\section{Materials and Methods:}

\section{System Setups:}

A system consisting of TM/CT domains of integrin $\alpha \operatorname{IIb} \beta 3$ (PDB: $2 \mathrm{KNC}(32)$ ) embedded in the plasma membrane, talin1 F2/F3 subdomains (PDB: 3IVF (20)), and kindlin2 FERM domain (PDB: 5XQ0 (33)) (ITK) was built using VMD. Two control systems in which integrin $\alpha \operatorname{Il} \beta 33$ was simulated in complex with either talin1 F2/F3 subdomains (IT) or kindlin2 FERM domain (IK) were also built. Two regions of the kindlin2 FERM domain are missing in solved crystal structure: residues 168-217 in the F1 subdomain and residues 337-512 in the F2 subdomain.

The three systems were solvated in explicit water using NMAD solvated package. The TIP3 water model was used for solvation (34). However, the water molecules inside the lipid membrane were removed. Then the systems were neutralized and ionized with $150 \mathrm{mM}$ of $\mathrm{KCl}$. The number of atoms reached 744946 in ITK, 556876 in IT, and 625742 in IK.

\section{MD simulations:}

We performed all-atom molecular dynamics (MD) simulation to investigate the conformational changes of integrin $\alpha \operatorname{IIb} \beta 3$ upon simultaneous interaction with talin1 and kindlin2 (ITK) and compared them to the two control simulations (IT and IK) (Fig. 1A). Molecular dynamics simulations were carried out using NAMD and CHARMM36 force field (35). Molecular visualization and analysis were performed using Visual Molecular Dynamics (VMD) package (36). Periodic boundary conditions were used in all three dimensions and a 2 fs time step was used in all simulations. The Langevin piston Nose-Hoover pressure control algorithm, and the Langevin damping thermostat for temperature control were used (35). Pressure was maintained at 1 bar and temperature at $310 \mathrm{~K}$ with a damping coefficient of 5/ps.

Each trial was initially minimized for 100,000 timesteps using the conjugate gradient and line search algorithm to relax the structures and remove all bad contacts. Following the minimization process, each configuration was equilibrated for $5 \mathrm{~ns}$ or longer until equilibrium was reached. Fully equilibrated structures were then used in the final production simulations that ran for 1000 ns for ITK and IT, and $760 \mathrm{~ns}$ for IK.

\section{H-bond calculations}

VMD hbonds plug-in (version 1.2) (36) were used to calculate the number of hydrogen bonds (hbonds) between regions of interest. The cutoff distance and angle were set to $4 \mathrm{~A}^{\circ}$ and 20 , respectively. The density- and time-dependent plots were all prepared in $\mathrm{R}$ matrixStats and gplot $(37,38)$.

\section{Principal component analysis (PCA)}

The PCA analysis was used to examine time evolution of the integrin structure along all trajectories and understand the conformational differences induced by cytoplasmic interactions. The principal components were defined as the orthogonal axes of maximal variance and 
identified by superpositioning the structures on the invariant core and calculating the variance using the tools introduced by the Bio3D package (39).

\section{Residue cross correlation}

To examine how the atomic fluctuations and displacements within the integrin heterodimer are correlated, we used the cross-correlation function in the Bio3D package (39). The matrix of all pairwise cross-correlations between residues were visualized using the dynamical crosscorrelation map.

\section{Cross correlation function}

The cross correlation function (CCF) in $\mathrm{R}$ was used to understand the relationship between two time series representing different features along the trajectories. The first argument was treated as the predictor (cause) or the second argument. The lag period indicates when the effect of a change in one feature is reflected in the other feature (39).

\section{Solvent accessible surface area (SASA)}

The solvent-accessible surface area of each molecule was calculated using the measure SASA command in VMD (36). This command

\section{Force distribution analysis}

To monitor changes in the internal forces of the integrin heterodimer upon cytoplasmic interactions, we performed Time-resolved Force Distribution Analysis (TRFDA) implemented in the GROMACS software package (40). Atomic pairwise forces were calculated for all residues of both integrin subunits and included only the non-bonded electrostatic interactions acting on each residue. The comparison between force propagation along the transmembrane domains of integrins indicated the allosteric signal propagation across the membrane. The punctual stress measured for alpha- and beta-subunits is the sum of absolute values of scalar pairwise forces exerted on each atom.

\section{Structural alignment}

To determine the changes in the conformation of kindlin and talin upon interactions with integrin, we compared the initial structures of these molecules with the final structures after $\sim 1000$ ns of simulation. The final structures of talin in the ITK or IT simulations were aligned to the initial structure of talin using the struct.aln function in the bio3D package in R (39). Similarly, the final structures of kindlin in the ITK or IK simulations were aligned to the initial kindlin structure. To quantify the distances between residues in the two aligned structures, a difference vector was calculated between the two structures using the dist.xyzfunction in R (39). 


\section{Results:}

To explore the role of kindlin in the activation of integrin as part of the integrin-mediated insideout signaling, we developed all-atomic microsecond-scale molecular dynamic simulations of $\alpha$ llb $\beta 3$ using an explicit lipid-water environment under three distinct scenarios, namely integrin in complex with talin1 F2-F3 subdomains (IT), with kindlin2 (IK), and with both talin and kindlin (ITK).

\section{The interactions of talin1 and kindlin2 with the $\mathrm{CT}$ of integrin $\alpha \operatorname{IIb} \beta 3$ regulate its conformation}

The mechanism of interaction of talin 1 and kindlin 2 with integrin $\beta 3$ can be characterized based on the conformational transitions of the integrin heterodimer. These conformational changes were determined by comparing the first and last frames of our microsecond molecular dynamics simulations, featuring identical starting conformation of integrin for all scenarios (Fig. 2A). The crossing angle between the transmembrane regions of integrin monomers was notably increased in IT simulations changing the shape to open scissors (see below for further details). Unexpectedly, the final shape of integrin in the ITK simulation was changed to closed scissors (Fig. 2A). No significant change in the crossing angle was observed in the IK simulation.

Conformational transitions of the integrin dimer in response to cytoplasmic interactions with talin1 and/or kindlin2 were quantified via a principal component analysis (PCA). For PCA calculations, the invariant core of the structure in each simulation was identified, the distribution of structures was extracted from each trajectory, and conformational differences were quantified for equivalent residues. The first two principal components accounted for over $50 \%$ of the variance in ITK and IK, while in IT this number reduced to $40 \%$. Since the contribution of all other principal components was relatively minimal, the structural distribution was projected in the PC1-PC2 space as shown in Fig. 2B. Three distinct clusters exist in IT and ITK, each representing structurally similar states, whereas only two are recognizable in IK. Moreover, final states of integrin in IT and ITK diverged significantly relative to the first frame.

Residue cross correlation analysis between all residue pairs of the integrin $\alpha \mathrm{IIb}$ and $\beta 3$ in ITK, IT, and IK was performed to examine how the fluctuations within the integrin dimer are correlated. Interestingly, we observed a coupling between the transmembrane regions of integrin $\alpha \mathrm{IIb}$ and $\beta 3$ (black boxes in Fig. 3C), which were dominantly correlated in ITK, but anticorrelated in IT. The level of residue correlations were notably lower in IK simulations resulting in a relatively lighter heat map (Fig. 3C). Residues on the left side of the boxed region are corresponding to near the outer membrane clasp (OMC) of the $\alpha$ subunit and were negatively correlated with the beta transmembrane region in ITK and IT, while this correlation was eliminated in IK.

In addition, we calculated the crossing angle between $\alpha$ IIb and $\beta 3$ helices $(\theta)$ as a function of time (Fig. 2D) to quantify the conformational changes of integrin. Specifically, we calculated the 
angle between the line crossing residues 967 to 979 in $\alpha \mathrm{IIb}$ and the line crossing residues 697 to 709 in $\beta 3$. As expected, an increase in crossing angle of $\beta 3$ in IT simulations was observed. Interestingly, in ITK, the $\theta$ angle starts from 40 and then approaches zero after $250 \mathrm{~ns}$ and $\alpha$ IIb and $\beta 3$ helices become almost parallel to each other. In the case of binding of kindlin 2 without talin1 (IK), our results showed a small angle change (10 degrees) in $\theta$, which is clearly in contrast with the cases of binding of talin1 that resulted in a noticeable change of $\theta$ (i.e., 30 degrees change in IT and 40 degrees change in ITK). 


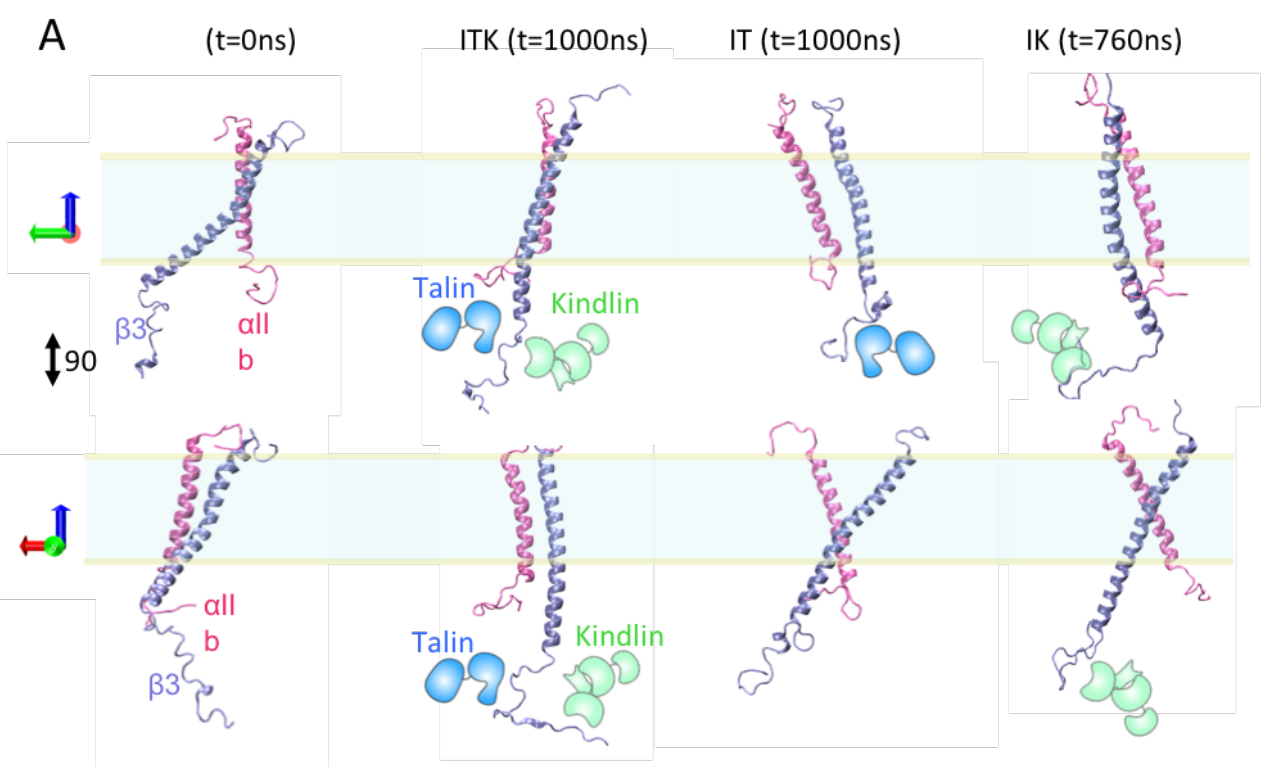

B

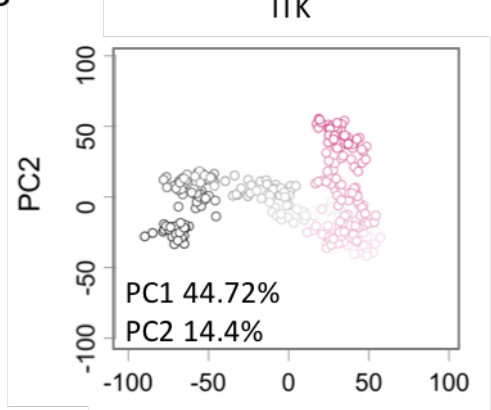

IT
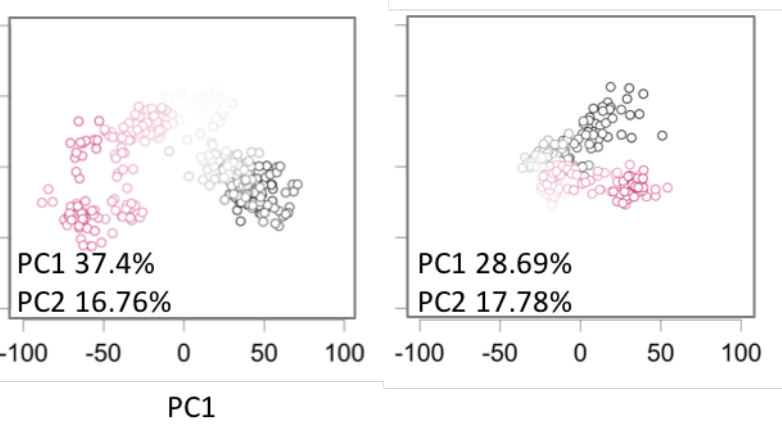

C
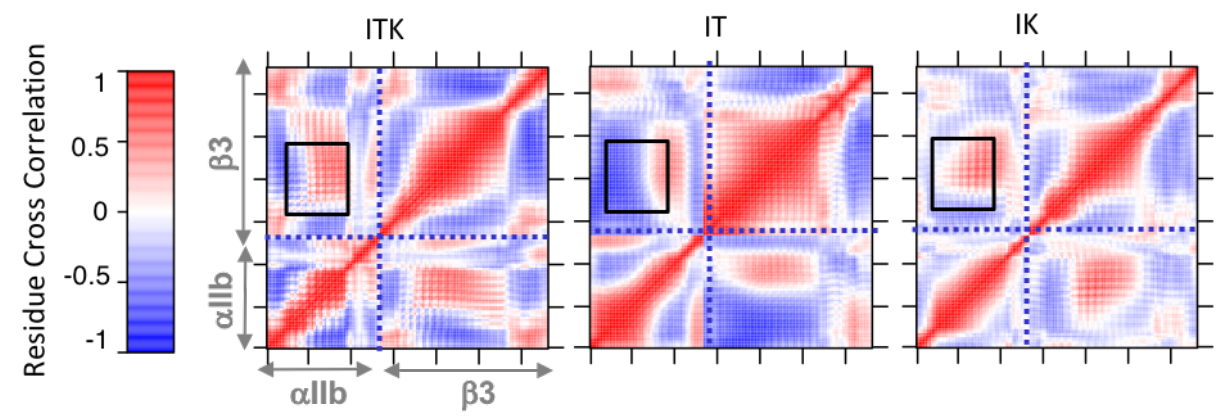

D
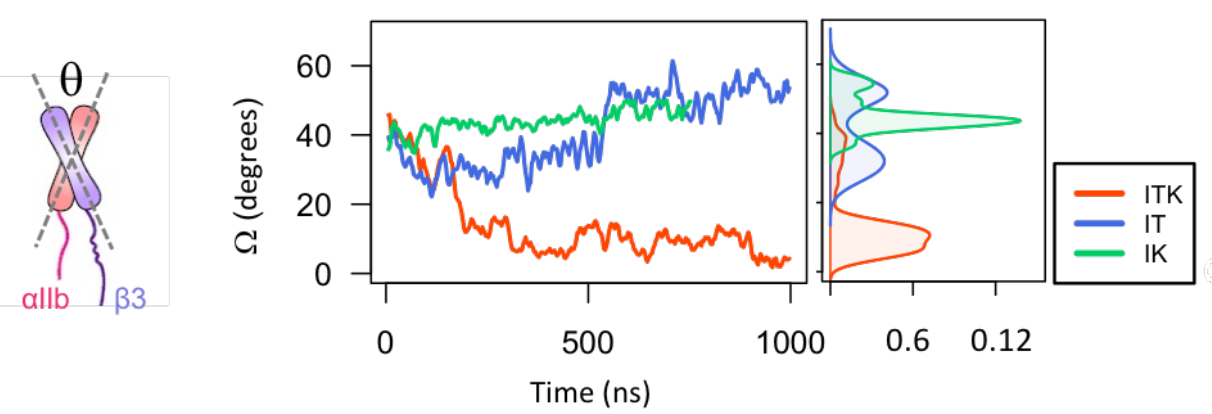


\section{Figure 2: Conformational changes of integrin aIIbb3}

(A) Schematics of talin and kindlin overlaid with the cartoon representations of the integrin $\alpha$ IIb (mauve) $\beta 3$ (iceblue) heterodimer in two different views (90 degrees) (left top and bottom). Snapshots of the trajectories of the integrin $\alpha \mathrm{IIb} \beta 3$ dimer for the last simulation frame of the ITK ( $\mathrm{t}=1000 \mathrm{~ns})$, IT ( $\mathrm{t}=1000 \mathrm{~ns})$, and IK ( $\mathrm{t}=760 \mathrm{~ns})$ simulations are shown. (B) The principal component analysis (PCA) of all trajectories. Each point is a structural state in the PC1-PC2 space. Points corresponding to the beginning of all trajectories are shown in red, middle frames are represented by smooth color change to white and end frames are illustrated in black. (C) Residue cross correlation heatmaps of the integrin $\alpha \mathrm{IIb}$ and $\beta 3$ helices averaged over simulation time. The $\alpha \mathrm{IIb}$ and $\beta 3$ regions are indicated on the heatmaps. Black boxes indicate the TM regions of integrin dimer. A representative heatmap is shown for each of the three ITK, IT, and IK simulations. (D) A schematic representation of the integrin dimer showing angle $\theta$ between the $\alpha \mathrm{IIb}$ and $\beta 3$ helices (right). The time plot and density plot of angle $\theta$ for the ITK, IT, and IK simulations are shown in red, blue, and green respectively.

\section{Interactions between $\alpha$ IIb and $\beta 3$ upon binding of talin 1 and kindlin 2}

In order to determine the conformational transition in integrin $\beta 3$ upon binding of talin 1 and kindlin2, we next analyzed the interaction energy between $\alpha \mathrm{IIb}$ and $\beta 3$ helices. Two interaction interfaces on the integrin $\alpha \operatorname{IIb} \beta 3 \mathrm{TM} / \mathrm{CT}$ domains are known to stabilize the resting conformation of the dimer: the inner membrane clasp (IMC) and the outer membrane clasp (OMC). IMC is characterized by the interactions between the highly conserved ${ }^{991}$ GFFKR $^{995}$ motif of $\alpha \mathrm{IIb}$ and $\mathrm{W} 715, \mathrm{~K} 716$, and $\mathrm{I} 719$ residues of $\beta 3$, and a salt bridge between residue R995 on $\alpha$ IIb and D723 on $\beta 3$ (Fig. 3A). The R995-E726 salt bridge is also known to be involved in maintaining integrin in an inactive conformation. OMC is defined as an interaction network between ${ }^{972} \mathrm{GXXXG}^{976}$ on $\alpha \mathrm{IIb}$ and $\mathrm{V} 700, \mathrm{M} 701$, and $\mathrm{I} 704$ residues on $\beta 3$, as well as associations between $\alpha \mathrm{IIb}{ }^{979} \mathrm{LL}^{980}$ and $\beta 3{ }^{705} \mathrm{LXXG}^{708}$ residues. Mutations in the residues involved in IMC or OMC result in disruption of interactions between $\alpha \mathrm{IIb}$ and $\beta 3$ helices leading to integrin activation $(6,19,28,41,42)$.

To examine whether the binding of kindlin2 or talin1 could disrupt the IMC and OMC interactions in our simulations, we calculated the non-bonded interaction energy between IMC and OMC regions over the course of simulation time (Fig. 3B). Our results showed that the nonbonded interaction energies of IMC and OMC decrease upon simultaneous binding of talin and kindlin (ITK simulation) and stabilizes after 250ns approximately zero in IMC and $-4 \mathrm{kcal} / \mathrm{mol}$ in OMC. In IT simulation, the nonbonded interaction energy in OMC approaches zero and remains zero after 500ns. On the other hand, IMC interactions in IT simulations switch between zero and $-100 \mathrm{kcal} / \mathrm{mol}$. In IK simulation, the level of OMC and IMC both remain stable at $-7 \mathrm{kcal} / \mathrm{mol}$, and $-120 \mathrm{kcal} / \mathrm{mol}$, respectively. These results indicate that kindlin2 and talin 1 cooperation can destabilize integrin $\alpha \mathrm{IIb} \beta 3$ dimer at IMC region and weaken their association in OMC region. Our results also suggest that kindlin2 alone is not sufficient to disrupt the IMC or OMC interactions.

Mechanical signal transmission entails dynamic force redistribution across the integrin $\alpha \operatorname{IIb} \beta 3$ molecule. To investigate how cytoplasmic interaction(s) with talin1 and/or kindlin2 allosterically impact the stress distribution within the transmembrane domain, especially near the IMC and 
OMC, we performed time-resolved force distribution analysis (FDA) as shown in Fig. 3D. The time-average of punctual stress of all integrin residues was used as a measure to compare force distribution patterns between simulations as shown in Fig. 3D, bars indicate standard deviation. Significant differences in the time-averaged punctual stress $(>80 \mathrm{KJ} / \mathrm{mol} \mathrm{nm})$ were almost exclusively observed in IK compared to ITK and IT. Specifically, time-averaged punctual stresses experienced in residues 704 and 705 (part of IMC), 706, 711, 715 (part of OMC), 717, 722,723 and 724 were remarkably higher in IK, while they were almost the same in IT and ITK. One exception was residue 726 for which time-averaged punctual stress in IT was higher than that in the ITK and IK simulations. Notably, residue 711 showed a most significantly different punctual stress within the IK simulation. 
A

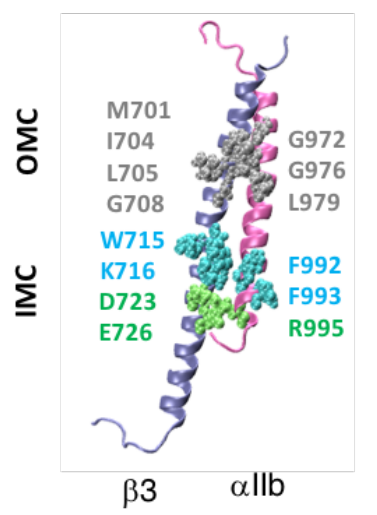

B

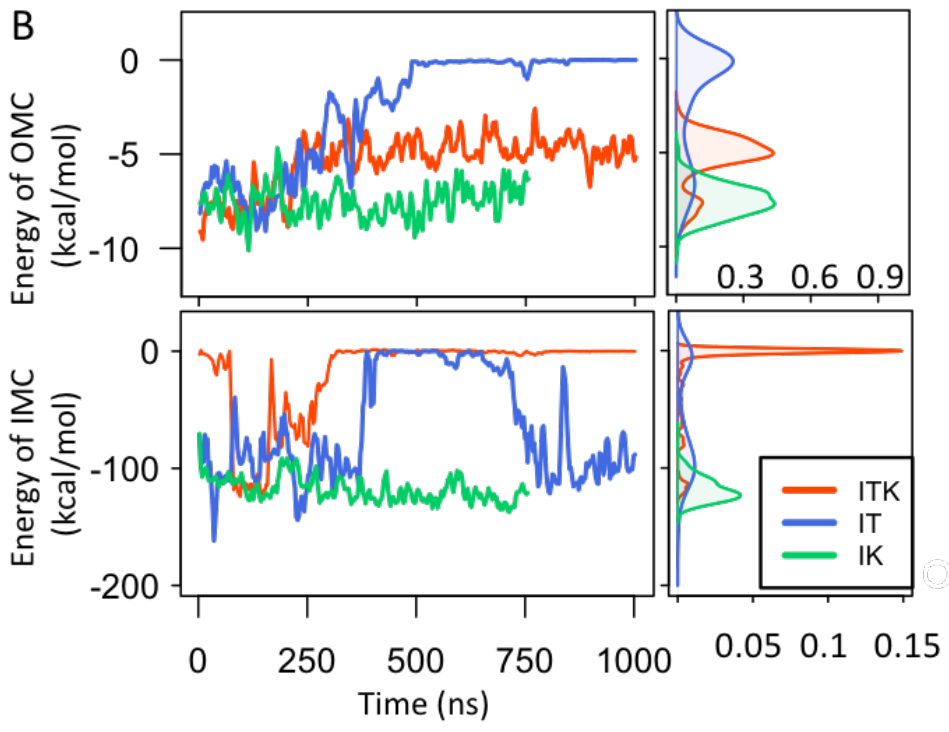

C

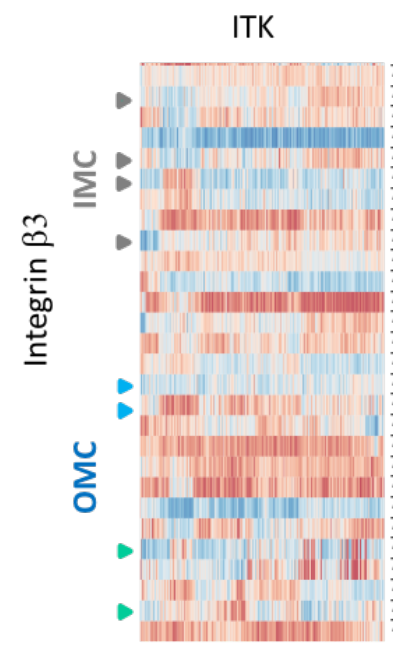

IT

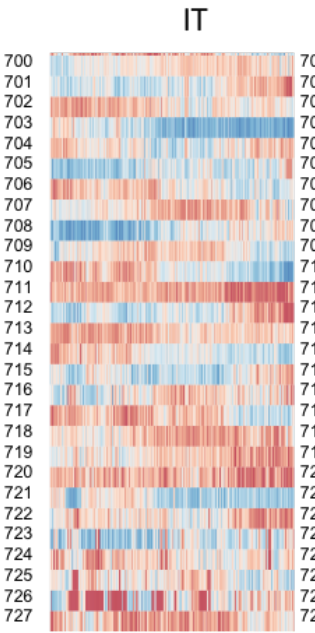

IK

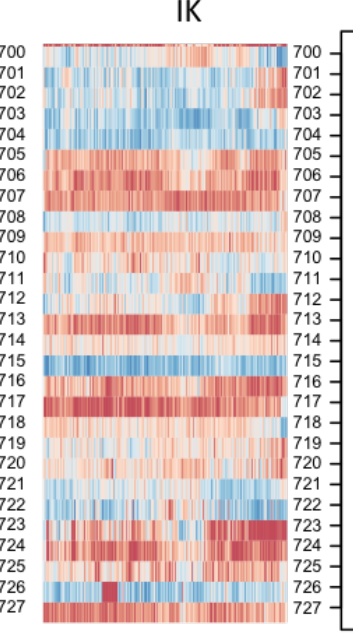

Average punctual stress $(\mathrm{KJ} / \mathrm{mol} \mathrm{nm})$
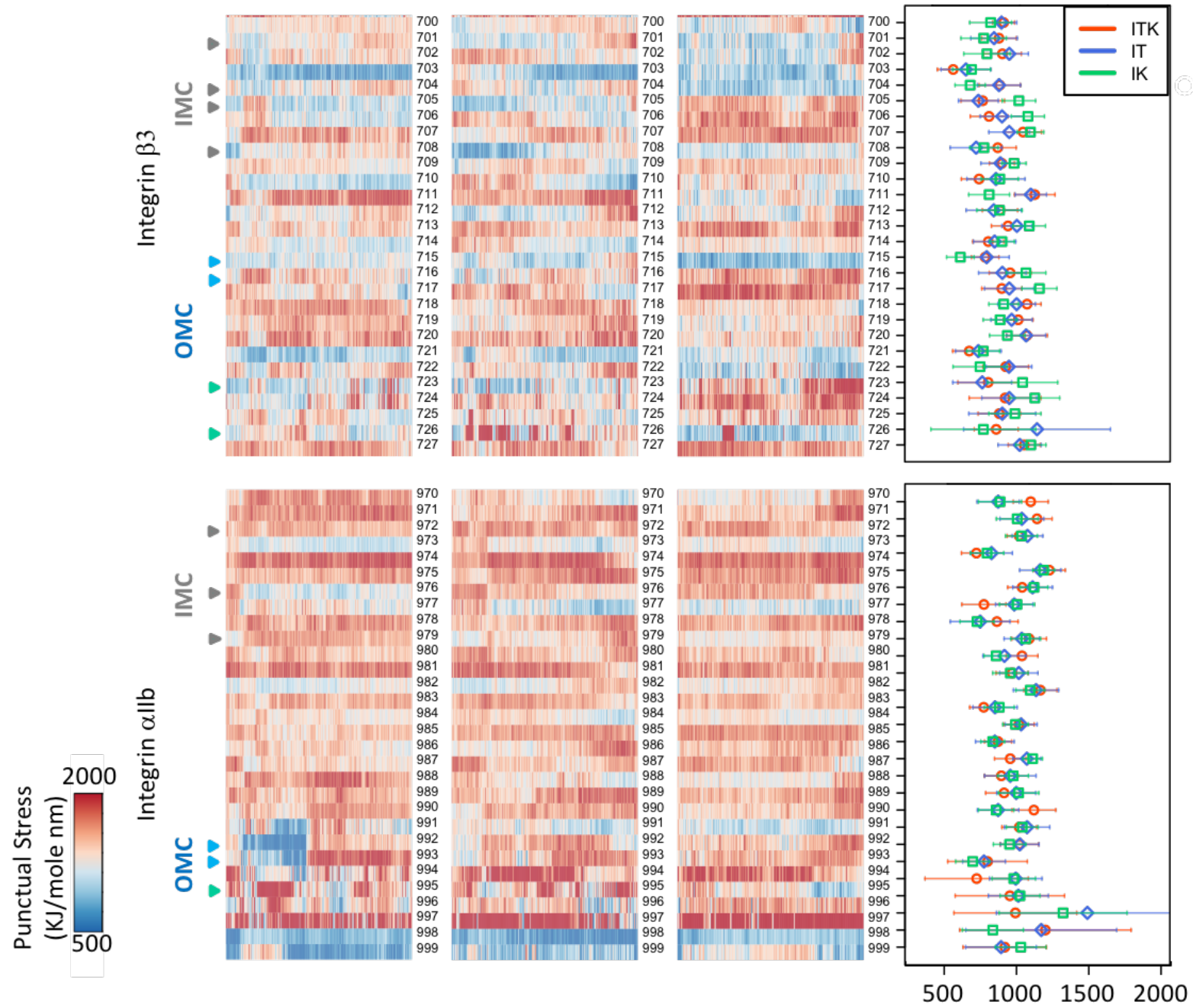
Figure 3: Interactions between $\alpha$ IIb and $\beta 3$ dimer

(A) Structure of the $\alpha \mathrm{IIb}$ (mauve) $\beta 3$ (iceblue) heterodimer. Interacting residues in the OMC region are shown in silver, in the IMC region in blue, and the $\alpha$ IIb R995 - $\beta 3$ D723/D726 salt bridges in green. (B) The time plot and density plot of energy of interactions between OMC (top) and IMC (bottom) residues are shown for the ITK, IT, and IK simulations in red, blue, and green, respectively. (C) Time-resolved force distribution analysis (FDA) and timeaveraged puntual stress within the integrin $\alpha \mathrm{IIb}$ and $\beta 3$ dimer were calculated. Representative heat maps of perresidue punctual stress values over simulation time for the integrin $\beta 3$ residues 700 to 727 and $\alpha$ IIb residues 971 to 996 are shown. A representative heatmap is shown for each of the three ITK, IT, and IK simulations. Arrows highlight the location of IMC and OMC residues, which show significant changes in punctual stresses under force.

\section{$\alpha$ IIb- $\beta 3$ angle change $(\theta)$ is correlated with OMC}

To understand whether changes in the angle ( $\theta$ ) between $\alpha \mathrm{IIb}$ and $\beta 3$ (Fig. 2D) was correlated with the interaction energies of IMC and OMC (Fig. 3B), we performed a cross correlation analysis using the cross correlation function for time series in R, as described in Methods (Fig. 4). This function computes the covariance between two time series up to a defined lag. The $\theta$ angle was not a significant predictor of IMC, most likely because IMC was either abruptly disrupted or remained stable throughout the simulation. This suggests that IMC disassociation is directly regulated by cytoplasmic interactions and not through angle change. In other words, interaction at the cytoplasmic region exerts tension that is transmitted by both bonded and nonbonded interactions across integrin residues towards the membrane, which eventually breaks IMC and allows angle change. Conversely, dynamics of OMC interaction was highly correlated with $\theta$ in ITK and IT. The reverse correlations of ITK and IT reflects opposite directions of angle change (Fig. 4). Since there is no significant angle change $(\theta)$ in the IK simulation, we see a minimal $\theta$-OMC correlation compared to ITK and IT.

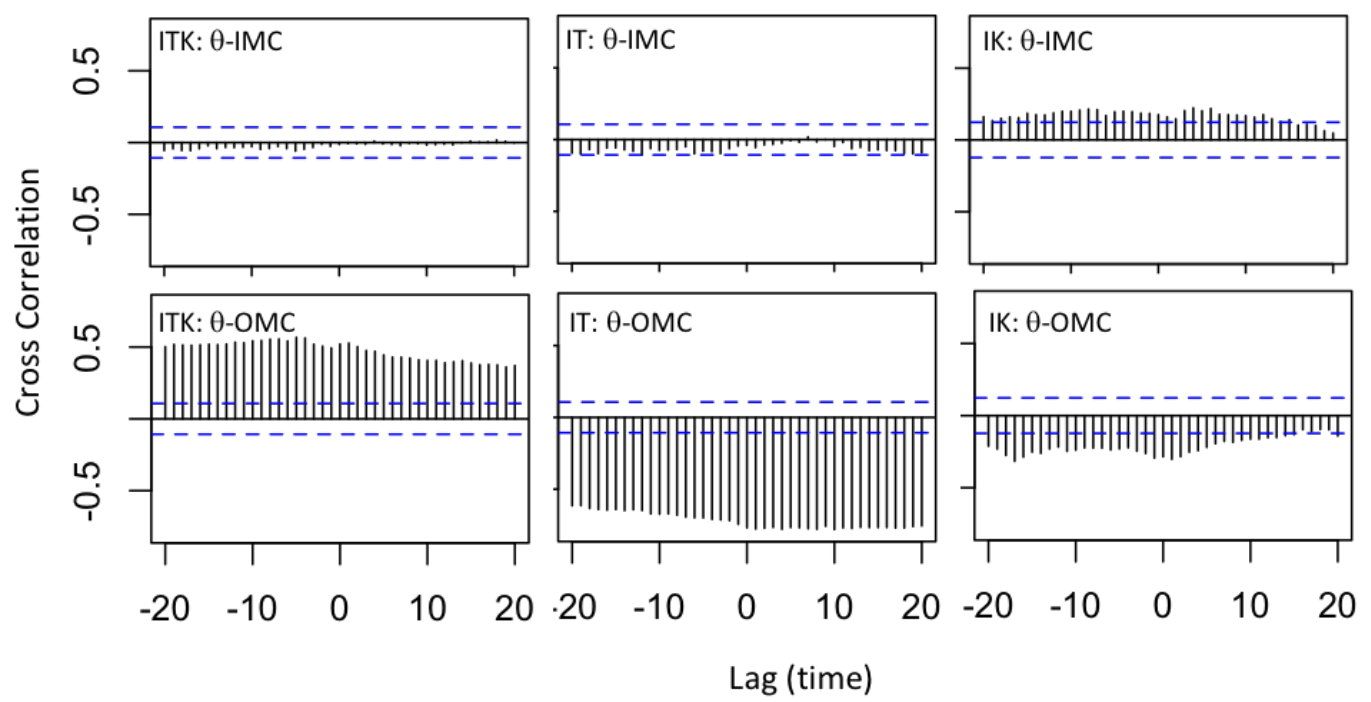

Figure 4: $\theta$ angle correlation with IMC and OMC

Cross correlation of IMC and angle changes of $\alpha \mathrm{IIb}$ and $\beta 3$ (top); and OMC and angle changes of $\alpha \mathrm{IIb}$ and $\beta 3$ (bottom) are shown. The horizontal blue lines are the approximate $95 \%$ confidence interval. Cross correlation plot is shown for each of the three ITK, IT, and IK simulations. High correlations exist between $\theta$ and OMC in ITK and IT, while the other correlations are negligible. 


\section{Talin1 binds more effectively/strongly to integrin $\alpha \operatorname{IIb} \beta 3$ in the presence of kindlin2}

Integrin $\beta 3$ tail contains 47 residues and two NxxY motifs. The first NPLY motif (membrane proximal) is the binding site for talin1 and the second NITY motif (membrane distal) is the kindlin2 binding site. Talin1 and kindlin2 can simultaneously bind to $\beta 3$ through their F3 domains and initiate the inside-out signaling (3). In order to understand how this inside-out signaling may be initiated by the cooperation between kindlin 2 and talin1, we investigated the molecular mechanisms of simultaneous binding of talin 1 and kindlin 2 to the short $\mathrm{CT}$ of $\beta 3$ and compared them with individual binding of integrin with talin 1 and kindlin 2 in our simulations. Specifically, we calculated non-bonded interaction energies between integrin $\beta 3$ and kindlin 2 or talin1, as well as solvent accessible surface areas of integrin $\beta 3 \mathrm{CT}$, talin1 and kindlin2.

We first calculated the non-bonded interaction energy between the CT of integrin $\beta 3$ and talin1 or kindlin2 F3 domains (Fig. 5A). Specifically, we calculated the interaction between residues 720 to 762 of $\beta 3$ and residues 569 to 680 of talin 1 or residues 560 to 680 of kindlin2. In the IT simulations, talin and $\beta 3$ maintain an average interaction energy of $105 \mathrm{kcal} / \mathrm{mol}$ (Fig. 5A top). However, talin 1 is able to bind much more strongly to integrin $\beta 3$ over time in ITK simulations. In this simulation, the nonbonded interaction energy between talin 1 and $\beta 3$ increases from 100 to $400 \mathrm{kcal} / \mathrm{mol}$ with an average energy of $222 \pm 94 \mathrm{kcal} / \mathrm{mol}$. Kindlin2, on the other hand (Fig. 5A bottom) binds more strongly to integrin $\beta 3$ in IK simulation with an interaction energy of $258 \pm 61$ $\mathrm{kcal} / \mathrm{mol}$, compared to $124 \pm 57 \mathrm{kcal} / \mathrm{mol}$ in ITK simulation.

Next, we calculated the solvent accessible surface area (SASA) of the integrin-binding-F3 subdomains of talin1 and kindlin2, and integrin $\beta 3$ CT (see Materials and Methods, Fig. 5B). Our results show that the SASA of talin1 is lower in ITK simulations $\left(5929 \pm 210 \AA^{2}\right)$ compared with IT simulation $\left(6034 \pm 163 \AA^{2}\right)$ (Fig. 5A top). The SASA of kindlin2 immediately drops in the first $250 \mathrm{~ns}$ of simulation in IK simulations. However, when talin1 is present, the SASA of kindlin2 fluctuates much more slowly and is much higher in value as shown in the density plots (Fig. 5B middle). The SASA of integrin CT is the lowest in ITK simulations compared to IT or IK simulations. The average values of $2575 \pm 189$ for ITK, $2698 \pm 221$ for IT, and $2805 \pm 158$ for IK suggests that both can bind to various regions on integrin and reduce its SASA (Fig. 5B bottom). Also, in IT simulation, the SASA of integrin peaks at a slightly lower value than IK simulation, indicating a closer contact between integrin and talin1, compared to integrin and kindlin2 in conditions where only one of them is present. Based on the interaction energies and SASA results, it is evident that talin1 binds more effectively in the presence of kindlin2, and kindlin2 can bind to integrin much more strongly in the absence of talin1.

The crystal structures of the kindlin2-integrin $\beta 3$ and talin1-integrin $\beta 3$ complexes revealed the hbond network between the molecules (33). To determine the stability of kindlin2 or talin1integrin complex, we calculated the number of hbonds between integrin CT - kindlin F3 and integrin CT - talin F3. Our results show that talin1 forms a slightly higher number of hbonds with 
integrin $\beta 3$ tail in the presence of kindlin2. However, kindlin2 can form a larger number of hbonds with integrin $\beta 3$ tail in the absence of talin1.

Finally, to determine whether the interaction energies between integrin $\alpha \operatorname{Ib} \beta 3 \mathrm{CT}$ and talin 1 or kindlin2 were directly correlated with the changes observed in the crossing angle between $\alpha$ IIb$\beta 3(\theta)$, we also calculated the cross correlation between these parameters. The interaction between talin 1 and integrin $\beta 3$ was promptly formed in both IT and ITK, while kindlin 2 binding was relatively gradual. Kindlin2 binding lagged the angle change in IK, while in ITK, it did not strongly correlate with $\theta$ change. In both IT and ITK, talin binding did not show a major correlation with the angle change mainly because talin1 interaction was stable throughout the simulations. This suggests that a stable binding with talin 1 is necessary for the subsequent changes in the integrin conformation. However, the role of kindlin2 is more complex as it may both directly and indirectly modulate integrin activation. 
A

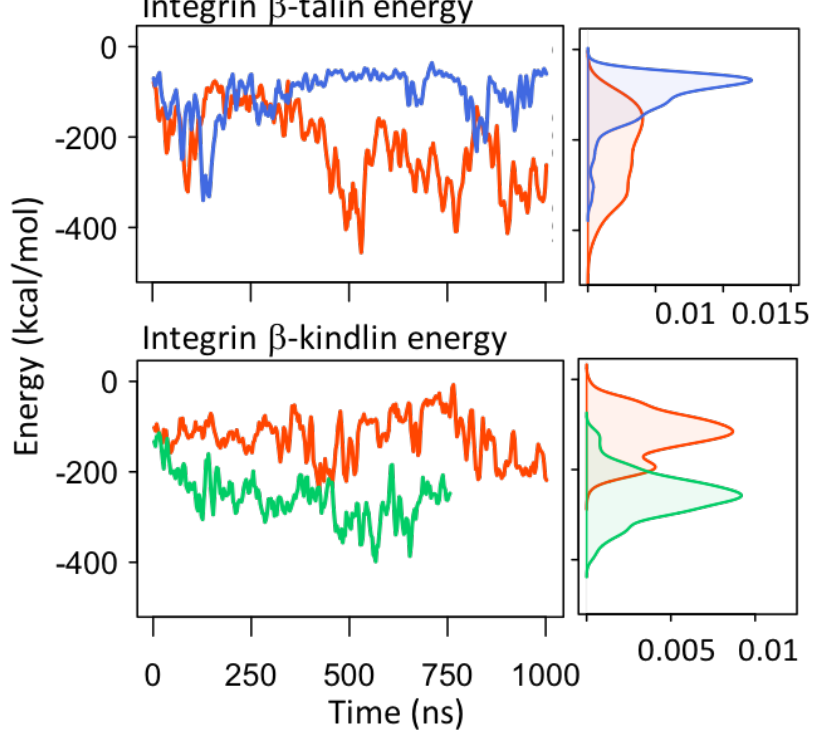

B

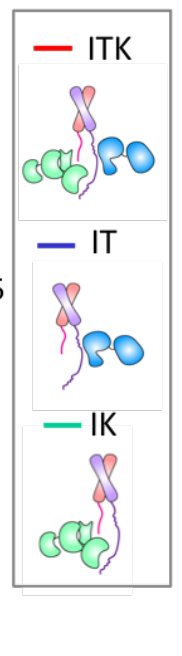

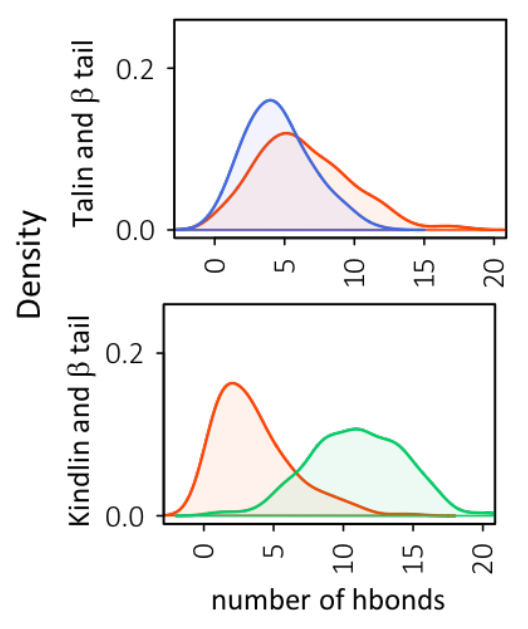

C

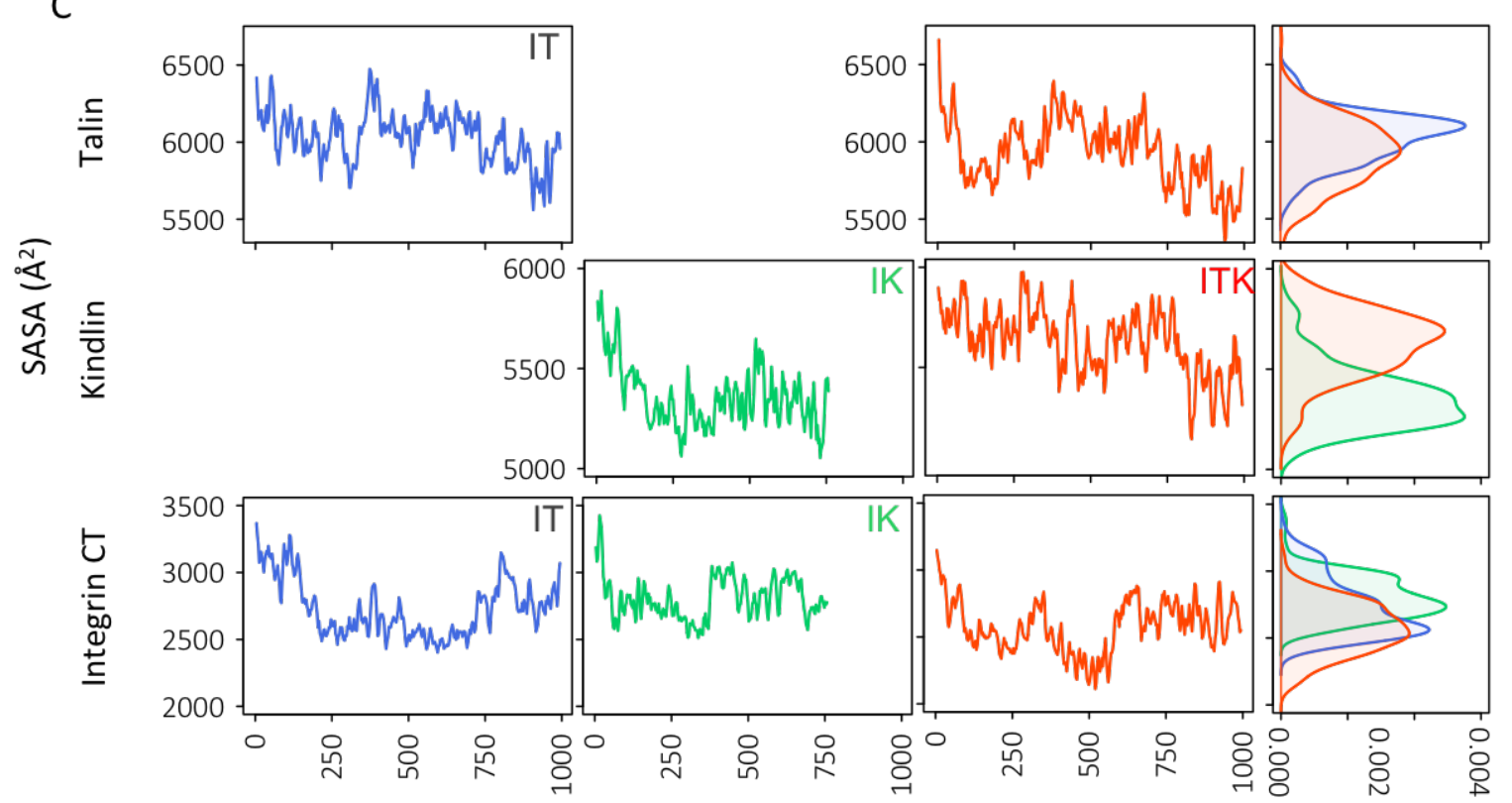

D

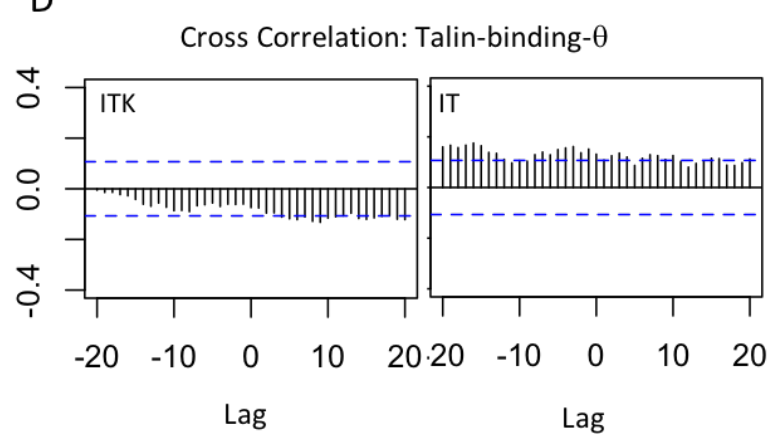

$E$

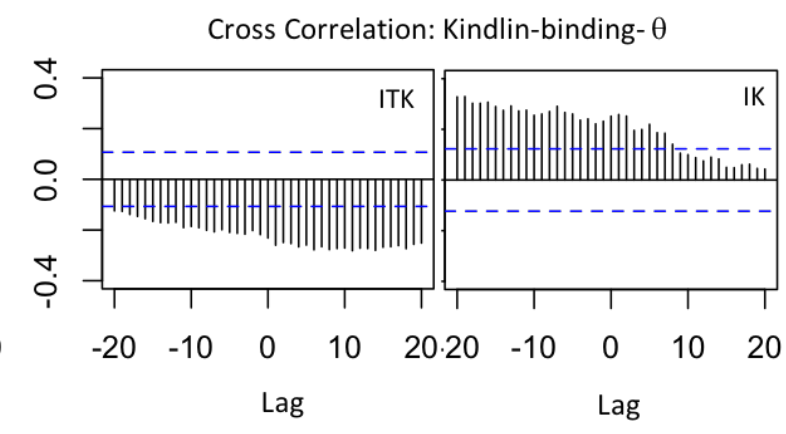


Figure 5: Integrin $\beta 3$-talin1 or kindlin2 binding

(A) The time and density plots of interaction energies between integrin $\beta 3 \mathrm{CT}$ and talin1 F3 domain (top) and integrin $\beta 3 \mathrm{CT}$ and kindlin2 F3 domain (bottom) are shown in three simulations of ITK, IT, and IK. (B) Density plot representations of the number of hbonds between integrin $\beta 3 \mathrm{CT}$ and talin $1 \mathrm{~F} 3$ domain (top) is shown for each of the ITK and IT simulations. The same plot is shown for kindlin2 (bottom) for each of the ITK and IK simulations. (C) Solvent accessible surface area (SASA) of talin1 (subdomain that binds integrin), in IT or ITK simulations (top). SASA of kindlin2 (subdomain that binds integrin), in IK or ITK simulations (middle). SASA of the CTs of integrin in IT, IK, or ITK2 simulations (bottom). (D) Cross correlation function between binding energy of talin1- integrin $\beta 3$ and $\theta$ in ITK and IT simulations are shown. (E) Cross correlation between binding energy of kindlin2 - integrin $\beta 3$ and $\theta$ in ITK and IK are shown (bottom).

\section{Force distribution analysis of integrin $\beta 3 \mathrm{CT}$}

We performed time-resolved force distribution analysis to monitor the dynamics of stress redistribution within the $\beta 3$ cytoplasmic region upon binding to talin1 and kindlin2. We have calculated the punctual stresses, scalar pairwise forces exerted on each atom, for the residues 728 to 762 of integrin $\beta 3$ cytoplasmic domain over time for both IT and ITK simulations (Fig. 6A). It can be seen from the heat map that the punctual stress fluctuates in most of the residues, except in residues 729 and 736, where the punctual stress remains high all the time both in IT and ITK.

Also, we have calculated the time-averaged punctual stress over the last $300 \mathrm{~ns}$ of the simulations (Fig. B). Different distinct regions can be identified on Fig. 6B and 6C based on whether the punctual stress of IT or ITK dominates. Time-averaged punctual stress is higher in residues 746 to 750 , which overlaps with the talin1 binding site (NPLY), in IT compared to ITK. Conversely, the kindlin2 binding site (NITY) consisting of residues 755 to 759 shows higher time-averaged punctual stress values in ITK compared to IT. This most likely indicates that direct binding with kindlin can locally increase stress levels. However, the region consisting of residues 738 to 744 exhibits higher average stress values in ITK compared to IT. We should note that in ITK, the stress value suddenly declines in residues 748 and 749 , while at the same time it increases in residues 737 and 739 .

Moreover, we see similar high stress values in the region consisting of residues 728 to 736 . Our analysis of binding interactions in this region revealed that these residues interact strongly with the membrane in both IT and ITK. On the other hand, the distance between the center of mass of talin F3 subdomain and integrin $\beta 3$ residues 728 to 736 decreases more significantly in ITK compared to IT (Fig. 6D). Therefore, the presence of kindlin resulted in significantly stronger interaction of talin with integrin $\beta 3$ MP region in ITK simulation. This is most probably an important factor in integrin activation as can disrupt IMC.

Furthermore, we studied the interactions of talin1 and kindlin2 with high-stress regions of integrin $\beta 3$ over the course of the ITK simulation. We observed three important interactions between the F3 subdomain of talin1 and the MP region of integrin $\beta 3$ (residues 723 to 736), namely, 1) talin K323- $\beta 3$ D723, 2) talin K321- $\beta 3$ E731, and 3) talin T370- $\beta 3$ A735 (Fig. 6E). In addition, interactions formed between the F3 subdomain of kindlin2 and the NITY motif of 
bioRxiv preprint doi: https://doi.org/10.1101/662163; this version posted June 6, 2019. The copyright holder for this preprint (which was not certified by peer review) is the author/funder. All rights reserved. No reuse allowed without permission.

integrin $\beta 3$ (residues 756 to 759). Specifically, residues N616/N618 and I621 of kindlin2 interacted with $\mathrm{I} 757$ and Y759 of integrin $\beta 3$, respectively, which remained stable for the last 50ns of the ITK simulation (Fig. 6F). 
A

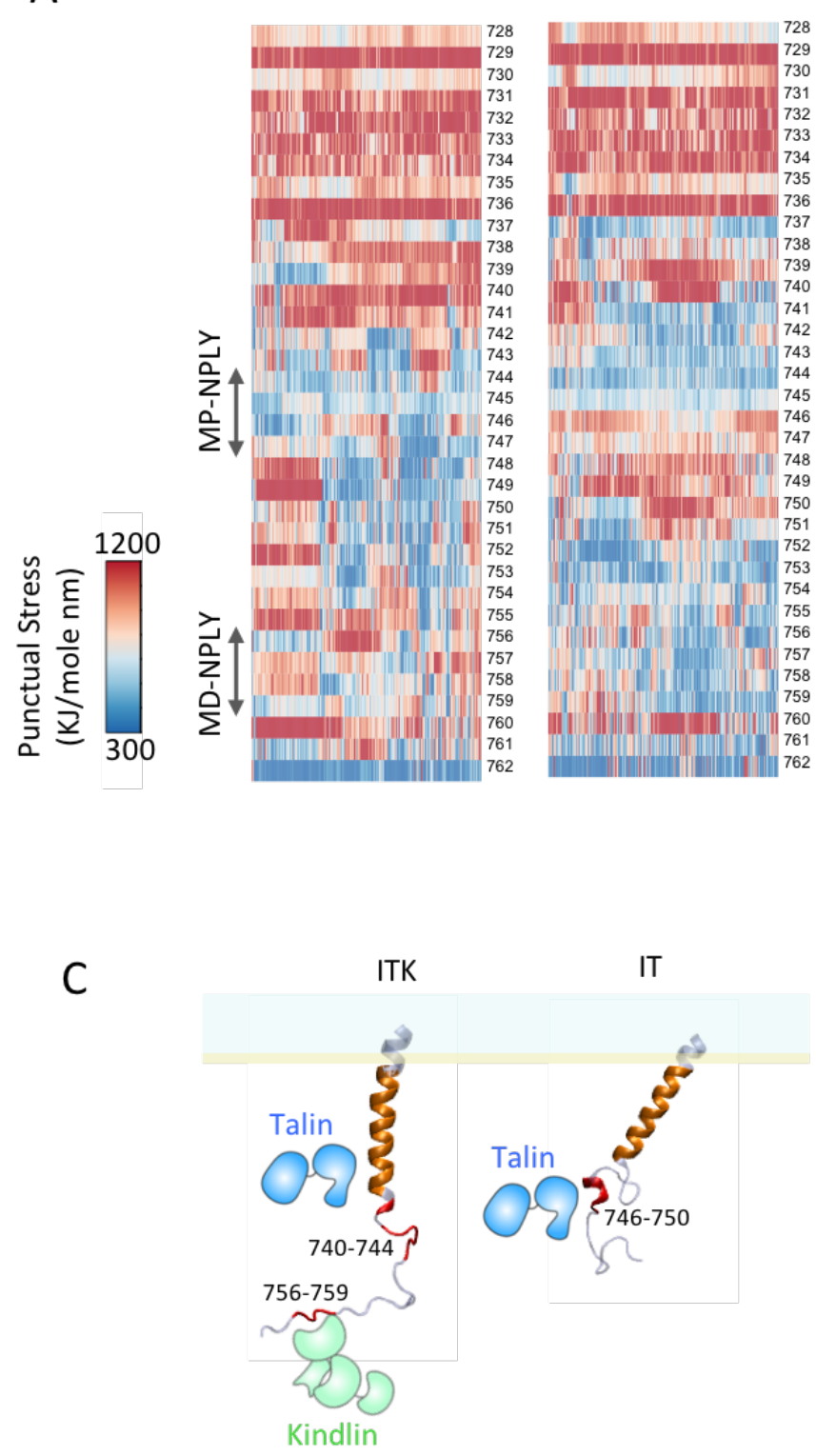

B

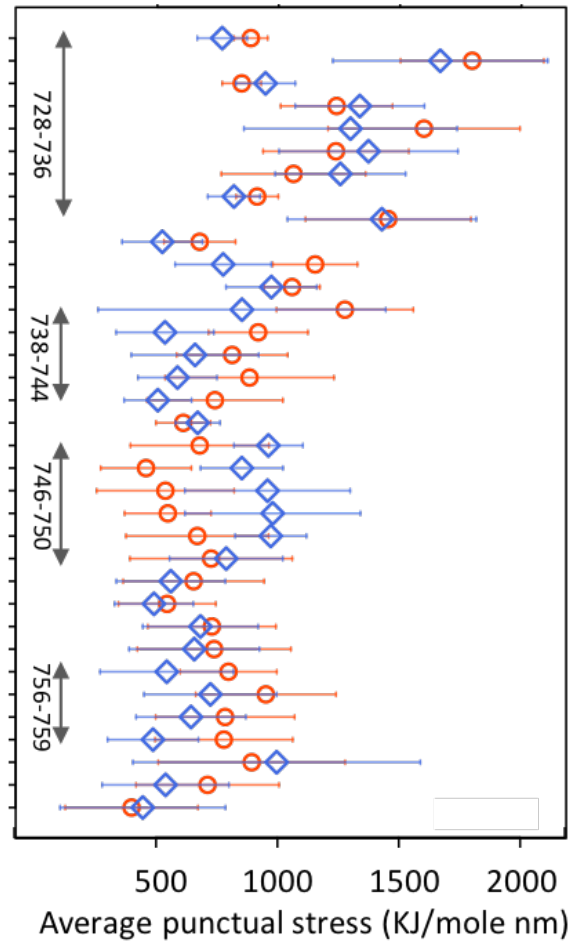

D

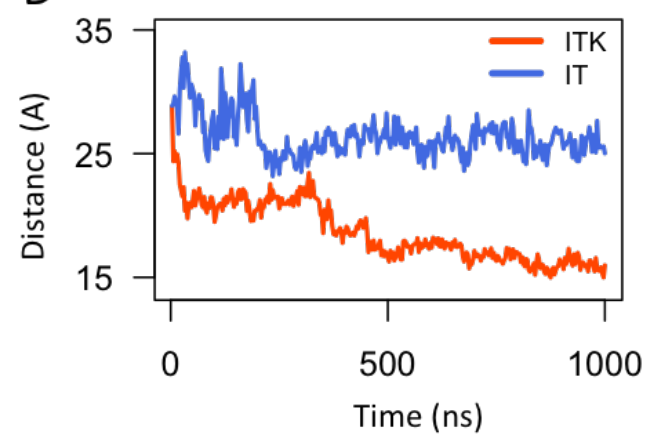

$\mathrm{F}$

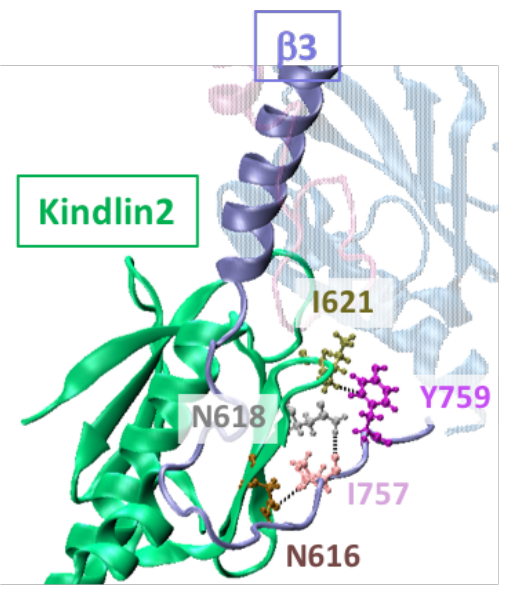


Figure 6: Force distribution analysis of integrin $\alpha \operatorname{IIb} \beta 3 \mathrm{CT}$

(A) TRFDA of integrin $\alpha$ IIb and $\beta 3$ subunits. Representative heat maps of per-residue punctual stress values over simulation time for the integrin $\beta 3$ cytoplasmic regions are shown. A representative plot is shown for each of the three ITK, IT, and IK simulations. (B) Schematic representations of the final structure of the integrin $\beta 3$ CT in ITK and IT simulations. Regions with high punctual stress values are shown in red and orange on $\beta 3$ CT. (C) The time plot of distance between the center of mass of residues 720 to 736 in integrin $\beta 3$ and talin 1 F3 subdomain are shown for the ITK and IT simulations in red, blue, respectively. (D) The time plot of distance between the center of mass of residues 720 to 736 in integrin $\beta 3$ and talin1 F3 subdomain are shown for the ITK and IT simulations in red, blue, respectively. (E) Strong interactions between talin1 F3 subdomain and integrin $\beta 3$ membrane proximal region in ITK simulation, mainly between residues K321, K323, and T370 on talin1, and residues D723, E731, and A735 on $\beta 3$. Hydrogen bonds are shown with dashed black lines. (F) Interactions between kindlin2 F3 subdomain and integrin $\beta 3$ NITY motif in ITK simulation, mainly between residues N616, N618, and TI621 on kindlin2, and residues $\mathrm{I} 757$ and Y759 on $\beta 3$. Hydrogen bonds are shown with dashed black lines.

\section{Distinct conformational changes of kindlin2 and talin1 in presence of each other}

To determine whether any conformational changes were induced in kindlin 2 or talin 1 by their mutual interactions with integrin $\alpha \operatorname{Ilb} \beta 3$, we performed structural alignments between the final structures of kindlin2 (at $\mathrm{t}=760 \mathrm{~ns}$ ) and talin1 (at $\mathrm{t}=1000 \mathrm{~ns}$ ) in each simulation, and their initial crystal structures. The structural alignments were performed using all sub-domains that were simulated (i.e. F2-F3 for talin1 and F0-F3 for kindlin2). However, for clarity, the alignments are shown separately for each sub-domain (Fig. 7). In order to quantify the deviation of each residue from its original position, the distance between residues of the initial and final structures were calculated after structural alignment was performed (Fig. 7). Our results show that the conformational changes observed in the F2 subdomain (residues 196 to 305) of talin1 are much higher in IT simulation compared with ITK simulation (Figure 7A). These conformational changes are mainly observed in two $\alpha$ helices ( $\sim$ residues 224 to 294$)$ in the four helix bundle of the F2 subdomain in IT simulation. The conformational changes observed in the F3 subdomain of talin1 were similar between IT and ITK conditions and mostly limited to the loop regions as expected. The structural alignment of kindlin2 showed significant conformational changes in the F0 subdomain in IK simulations, and F3 in ITK simulations, whereas the F1 and F2 subdomains showed very small changes in both simulations. Previous work showed that the F0 subdomain of kindlin2 is highly dynamic and moves independently of F1-F3 subdomains which could be a reason for the difference in the conformational state of F0 between different simulations. On the other hand, the conformational changes in F3 were seen in integrin binding regions (i.e. residues 590 to 656 ) and are likely attributed to the distinct interactions of kindlin 2 with integrin $\beta 3$ in IK vs ITK simulations. These results suggest that conformational changes are induced in talin 1 and kindlin2 upon interactions with integrin $\beta 3$, and that these changes are distinct when both molecules are present. 
A
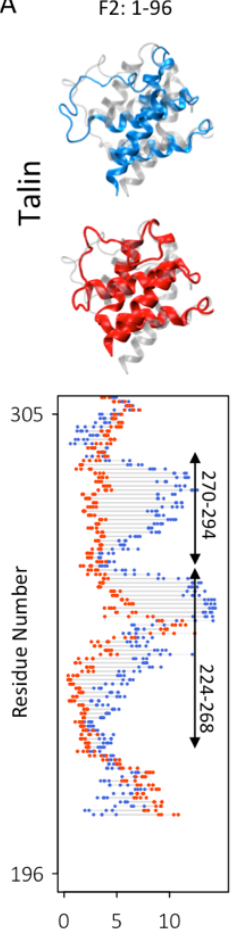
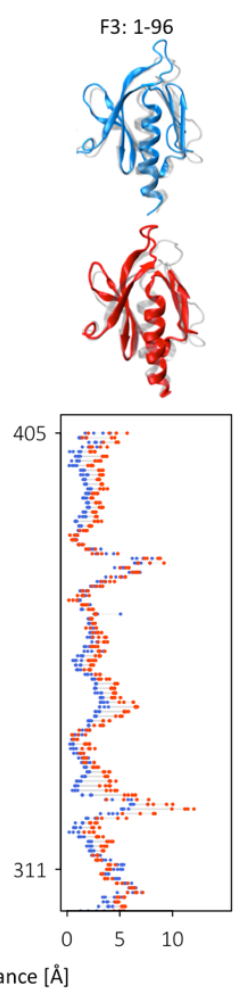

B
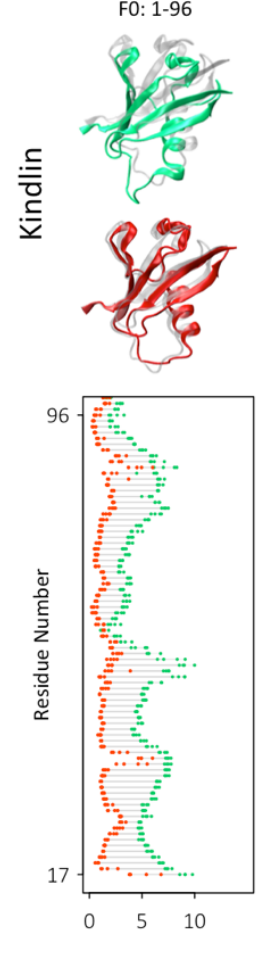

F1: 97-276
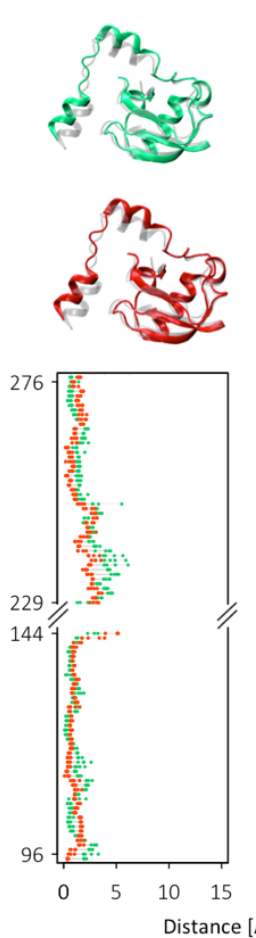

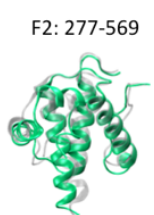

F3: 570 to 680
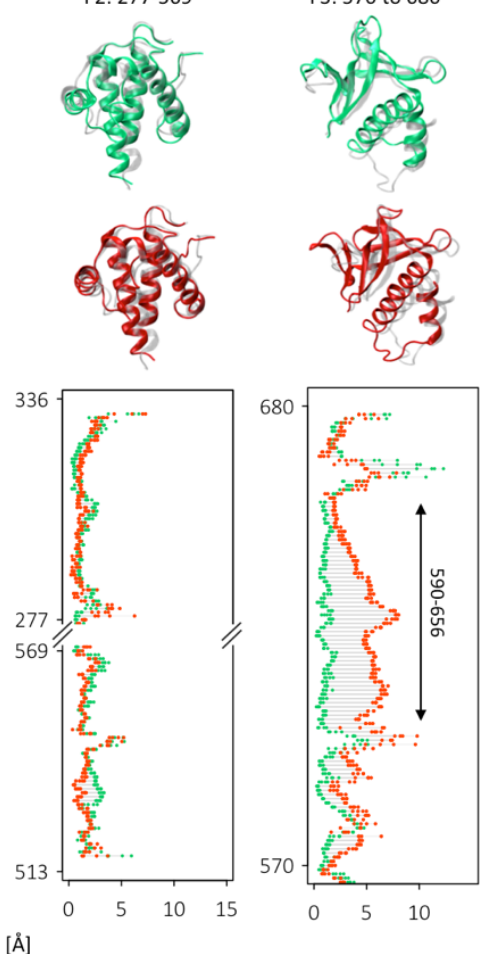

Figure 7: Kindlin2 and talin1 structural alignment

(A) Final frames of talin1 in IT (blue) and ITK (red) were aligned to initial pdb of talin1 (transparent). The distance shows the 3D distance between each residue in the two structures after alignment. (B) Final frames of kindlin2 in IK (green) and ITK (red) were aligned to the initial pdb of kindlin2 (transparent) (by RMSD minimization methods). The distance shows the 3D distance between each residue in the two structures after structural alignment. 


\section{Discussion:}

Integrin activation is central to a variety of cell functions, including cell-matrix adhesion, cell migration, and apoptosis. Kindlin and talin are both cytoplasmic proteins that directly interact with integrin tail and induce a high-affinity state of integrin for extracellular ligands (3). Although talin can induce conformational changes in integrin, it has been shown that talin alone is not sufficient for the activation of integrin and kindlin also plays a key role in this process $(14,17)$. While molecular mechanisms of talin-mediated integrin activation have been extensively studied, it is not yet clear how kindlin interferes with this process. Kindlin dysfunction has been associated with a number of human diseases, which are likely linked to deficiency in integrin activation; thus knowing the molecular mechanism of kindlin-mediated integrin activation has potential impacts in therapeutic purposes. Here, we investigated the interplay between talin and kindlin in integrin activation using microsecond long molecular dynamics simulations (Fig. 1).

Previous studies have shown that kindlin binding to integrin is not sufficient but is required for triggering integrin activation $(6,13,15)$. However, the mechanisms of kindlin's involvement remain elusive, in particular it is still not known whether kindlin can induce conformational changes to the integrin heterodimer. To address these questions and provide molecular details of the interaction among kindlin and talin with integrin, we developed all-atom microsecond-scale MD simulations of $\alpha$ llb $\beta 3$ using an explicit lipid-water environment under three distinct scenarios, namely integrin in complex with talin1 (IT), kindlin2 (IK), and both talin and kindlin (ITK). Our control simulations featuring integrin $\alpha \mathrm{IIb} \beta 3$ and kindlin2 (IK) showed that the main interactions that keep integrin in an inactive state, namely the IMC and OMC, remained stable over the course of $750 \mathrm{~ns}$ of the simulation when only kindlin is present (Fig. 3B). This further confirmed that kindlin binding is insufficient for unclasping cytoplasm and transmembrane regions of $\alpha \operatorname{IIb} \beta 3$.

Interestingly, our simulations showed that kindlin 2 binds to the integrin $\beta 3$ tail more strongly in the absence of talin1, whereas talin1 binding to integrin is reinforced by kindlin2 (Fig. 5). Talin1 interaction was particularly enhanced with the membrane proximal region of integrin $\beta 3$ (residues 720 to 736), while its interaction with the membrane MP NPXY motif was gradually weakened over time. Therefore, kindlin2 physically pushes talin1 towards the membrane resulting in a more effective disruption of the IMC. This way, kindlin2 modifies the activation pathway by positioning talin 1 more upward and closer to the membrane, resulting in stronger binding and a complete IMC disruption. Also, kindlin2 decreased the intradomain conformational changes of talin1, especially in F2 subdomain, which promoted both talin1-lipid and talin1-integrin $\beta 3 \mathrm{CT}$ interactions (Fig. 7A). Therefore, we suggest that the strength of kindlin2 binding to integrin does not necessarily correlate with activation, while stronger talin1 association can expedite the activation process. This further confirms the indirect role of kindlin2 as a cooperative partner for talin 1 rather than a direct activator of integrin $\alpha \operatorname{IIb} \beta 3$. It should be 
noted that even though we did not observe a strong interaction between talin1 and membrane proximal region of $\beta 3$ in IT simulations, it is quite conceivable that even without kindlin2's assistance, talin 1 can eventually bind to the proximal region of integrin tail in a longer timescale. On the other hand, the stronger binding of kindlin 2 to integrin in the absence of talin 1 observed in our simulations may have implications in talin-independent roles of kindlin in the formation and maturation of focal adhesions. Theodosiou et al. showed that subsequent to the cooperative activation of integrin by talin and kindlin2, kindlin2 initiates a talin-independent signaling pathway at new adhesion sites by binding to other molecules such as paxillin to induce cell spreading (15). Based on previous studies and our results, we propose that kindlin cooperates with talin to activate integrins by enhancing talin's interactions with the CT of integrin. On the other hand, kindlin can bind more strongly to integrin in the absence of talin to perform talinindependent signaling subsequent to integrin activation (15).

It is now widely accepted that the inner membrane clasp (IMC) and outer membrane clasp (OMC) between integrin subunits maintains the resting state of integrin and unclasping it triggers the inside-out signaling (6). In the IMC, R995 of beta-integrin forms two salt bridges with D723 and E726 on alpha-integrin, however, it has been shown that disrupting the R995-D723 interaction is most critical for integrin activation (6). Our simulations indicated that talin1 disrupts R995-D723 interaction in both presence or absence of kindlin2 (Fig. S1), however, kindlin2 is also required for dissociating R995-E726 within $1 \mu$ s of simulation (Fig. 3B).

Conversely, the OMC was destabilized in both cases (IT and ITK), causing significant conformational changes in the transmembrane region of integrin $\alpha \operatorname{Ilb} \beta 3$ (Fig. 2 and 3). The destabilization of OMC is highly correlated with the dramatic angle change between $\alpha$ IIb and $\beta 3$ $(\theta)$ in both IT and ITK (Fig. 4). We noticed that the angle change in ITK was in reverse direction relative to that in IT, i.e., decreasing angle in ITK and increasing angle in IT (see Fig. 2). Previous studies suggested that talin-mediated integrin activation involved an increase in the $\alpha I I b-\beta 3$ crossing angle and subsequent separation of the transmembrane domains $(7,27)$. However, recent studies showed that transmembrane domains of integrin subunits were close to each other in all conformational snapshots including active, inactive and intermediate states, contradicting the notion of chain separation as an indicator of integrin activation $(28,43)$. Since the angle change in ITK led to disruption of the IMC and OMC interactions, we propose that the degree of angle change is more pivotal in integrin activation than the direction of such change. This is most likely because talin1 and kindlin2 eliminate the inhibitory effects of IMC and OMC, which allows for mechanical signals to be transmitted across the membrane, without complete separation of the transmembrane regions. This is also demonstrated by changes in the force distribution patterns of the transmembrane domains of integrin in both IT and ITK simulations (Fig. 3). Furthermore, we showed previously that residue A711 in $\beta 3$ helix is key for signal transmission across the transmembrane domain of integrin and an A711P mutation can disrupt integrin activation (44). Our FDA analysis also indicated that A711 bears high forces in both IT and ITK but not in IK (Figure 3), implying that signal transmission is activated upon IMC 
disruption. Overall, our study suggests that not only both talin 1 and kindlin 2 are essential for efficiently initiating the activation process, but also kindlin2 may modify the molecular mechanism of inside-out signaling at the integrin allbb3 tail, which eventually leads to the opening of the integrin ectodomain.

Although not studied in this work, it is important to note that kindlin may have several other roles in integrin activation and the formation of focal adhesions. For example, one possible role of kindlin in integrin activation may be preventing the association of cytoplasmic inhibitors of integrin activation such as filamin or $\alpha$-actinin to the integrin tail. Also, kindlin may be involved in integrin clustering through dimerization (31). Moreover, kindlin binding to actin can enhance cytoskeletal coupling to the membrane. Therefore, simultaneous binding of kindlin and talin to the integrin $\beta$ increases the overall force applied to the integrin tail, which may facilitate focal adhesion formation and maturation.

Taken together, our results shed light on the molecular mechanisms by which kindlin2 cooperates with talin 1 in integrin $\alpha \operatorname{IIb} \beta 3$ activation. This is the first computational study, to the best of our knowledge, that models the interplay between kindlin 2 with talin 1 in promoting inside-out signaling through integrin binding. 


\section{Author Contributions}

All authors contributed to the design and implementation of the project and the computational experiments. All authors provided critical feedback in all steps of the project, which shaped the final research. ZH conducted the molecular dynamics simulations with inputs from HS and ZJ. All authors were involved in post-processing and analysis of the data and writing of the manuscript. MRKM supervised the project and contributed materials and analysis tools.

\section{Acknowledgments}

We acknowledge fruitful conversations with Mehrdad Mehrbod in the early stages of this research. This research used resources of the National Energy Research Scientific Computing (NERSC) Center, a Department of Energy Office of Science user facility supported by the Office of Science of the United States Department of Energy under contract no. DE-AC02-05CH11231.

\section{References:}

1. Humphries JD, Chastney MR, Askari JA, Humphries MJ. Signal transduction via integrin adhesion complexes. Curr Opin Cell Biol [Internet]. 2019;56:14-21. Available from: https://doi.org/10.1016/j.ceb.2018.08.004

2. Kim M, Carman C V, Springer TA. Bidirectional Transmembrane Signaling by Cytoplasmic Domain Separation in Integrins. Science (80- ) [Internet]. 2003 Sep 19 [cited 2019 May 20];301(5640):1720-5. Available from:

http://www.ncbi.nlm.nih.gov/pubmed/14500982

3. Luo B-H, Carman C V, Springer TA. Structural basis of integrin regulation and signaling. Annu Rev Immunol [Internet]. 2007 [cited 2019 May 20];25:619-47. Available from: http://www.ncbi.nlm.nih.gov/pubmed/17201681

4. Xiong J-P, Stehle T, Goodman SL, Arnaout MA. New insights into the structural basis of integrin activation. Blood [Internet]. 2003 Apr 24 [cited 2019 May 20];102(4):1155-9. Available from: http://www.ncbi.nlm.nih.gov/pubmed/12714499

5. Nieswandt B, Varga-Szabo D, Elvers M. Integrins in platelet activation. J Thromb Haemost [Internet]. 2009 Jul [cited 2015 Feb 17];7 Suppl 1:206-9. Available from: http://www.ncbi.nlm.nih.gov/pubmed/19630801

6. $\quad$ Bledzka K, Qin J, Plow EF. Integrin $\alpha I I b \beta 3$. Platelets. 2019;227-41.

7. Mehrbod M, Trisno S, Mofrad MRK. On the activation of integrin $\alpha$ IIb $\beta 3$ : outside-in and inside-out pathways. Biophys J [Internet]. 2013 Sep 17 [cited 2015 Jan 15];105(6):130415. Available from: http://www.cell.com/article/S0006349513009181/fulltext

8. Jahed Z, Shams H, Mehrbod M, Mofrad MRK. Mechanotransduction pathways linking the extracellular matrix to the nucleus. Int Rev Cell Mol Biol [Internet]. 2014 Jan [cited 2015 Feb 18];310:171-220. Available from: http://www.ncbi.nlm.nih.gov/pubmed/24725427

9. Ye F, Kim C, Ginsberg MH. Reconstruction of integrin activation. Blood. 2012;119(1):26-33.

10. Sun Z, Costell M, Fässler R. Integrin activation by talin, kindlin and mechanical forces. Nat Cell Biol [Internet]. 2019;21(1):25-31. Available from: http://dx.doi.org/10.1038/s41556-018-0234-9 
11. Ye F, Hu G, Taylor D, Ratnikov B, Bobkov AA, McLean MA, et al. Recreation of the terminal events in physiological integrin activation. J Cell Biol [Internet]. 2010 Jan 11 [cited 2019 May 20];188(1):157-73. Available from: http://www.ncbi.nlm.nih.gov/pubmed/20048261

12. Shattil SJ, Kim C, Ginsberg MH. The final steps of integrin activation: the end game. Nat Rev Mol Cell Biol [Internet]. 2010 Apr 1 [cited 2018 May 22];11(4):288-300. Available from: http://www.ncbi.nlm.nih.gov/pubmed/20308986

13. Plow EF, Qin J. The Kindlin Family of Adapter Proteins. Circ Res [Internet]. 2019 Jan 18 [cited 2019 May 8];124(2):202-4. Available from: https://www.ahajournals.org/doi/10.1161/CIRCRESAHA.118.314362

14. Ma Y-Q, Qin J, Wu C, Plow EF. Kindlin-2 (Mig-2): a co-activator of beta3 integrins. J Cell Biol [Internet]. 2008 May 5 [cited 2019 May 20];181(3):439-46. Available from: http://www.ncbi.nlm.nih.gov/pubmed/18458155

15. Theodosiou M, Widmaier M, Böttcher RT, Rognoni E, Veelders M, Bharadwaj M, et al. Kindlin-2 cooperates with talin to activate integrins and induces cell spreading by directly binding paxillin. Elife. 2016;5:1-24.

16. Kammerer P, Aretz J, Fässler R. Lucky kindlin: A cloverleaf at the integrin tail. Proc Natl Acad Sci U S A [Internet]. 2017 Aug 29 [cited 2018 May 22];114(35):9234-6. Available from: http://www.ncbi.nlm.nih.gov/pubmed/28830996

17. Plow EF, Qin J, Byzova T. Kindling the flame of integrin activation and function with kindlins. Curr Opin Hematol [Internet]. 2009 Sep [cited 2019 May 20];16(5):323-8. Available from: http://www.ncbi.nlm.nih.gov/pubmed/19553810

18. Moser M, Nieswandt B, Ussar S, Pozgajova M, Fässler R. Kindlin-3 is essential for integrin activation and platelet aggregation. Nat Med. 2008;14(3):325-30.

19. Ye F, Snider AK, Ginsberg MH. Talin and kindlin: The one-two punch in integrin activation. Front Med China. 2014;8(1):6-16.

20. Elliott PR, Goult BT, Kopp PM, Bate N, Grossmann JG, Roberts GCK, et al. The Structure of the Talin Head Reveals a Novel Extended Conformation of the FERM Domain. Structure [Internet]. 2010 Oct 13 [cited 2019 May 20];18(10):1289-99. Available from:

https://www.sciencedirect.com/science/article/pii/S096921261000300X?via\%3Dihub

21. Bledzka K, Liu J, Xu Z, Dhanuja Perera H, Yadav SP, Bialkowska K, et al. Spatial coordination of kindlin-2 with talin head domain in interaction with integrin $\beta$ cytoplasmic tails. J Biol Chem. 2012;287(29):24585-94.

22. Kalli AC, Campbell ID, Sansom MSP. Conformational Changes in Talin on Binding to Anionic Phospholipid Membranes Facilitate Signaling by Integrin Transmembrane Helices. PLoS Comput Biol. 2013;9(10).

23. Lau TL, Kim C, Ginsberg MH, Ulmer TS. The structure of the integrin ailbB3 transmembrane complex explains integrin transmembrane signalling. EMBO J. 2009;28(9):1351-61.

24. Calderwood DA, Campbell ID, Critchley DR. Talins and kindlins: Partners in integrinmediated adhesion. Nat Rev Mol Cell Biol. 2013;14(8):503-17.

25. Kurtz L, Kao L, Newman D, Kurtz I, Zhu Q. Integrin $\alpha \operatorname{IIb} \beta 3$ inside-out activation: An in situ conformational analysis reveals a new mechanism. J Biol Chem. 2012;287(27):23255-625.

26. Ginsberg MH. BMB-47-655.pdf. 2014;47(November):655-9. 
27. Kim C, Ye F, Hu X, Ginsberg MH. Talin activates integrins by altering the topology of the $\beta$ transmembrane domain. J Cell Biol. 2012;197(5):605-11.

28. Provasi D, Negri A, Coller BS, Filizola M. Talin-driven inside-out activation mechanism of platelet $\alpha \mathrm{IIb} \beta 3$ integrin probed by multimicrosecond, all-atom molecular dynamics simulations. Proteins Struct Funct Bioinforma. 2014;82(12):3231-40.

29. Bidone TC, Polley A, Jin J, Driscoll T, Iwamoto D V., Calderwood DA, et al. CoarseGrained Simulation of Full-Length Integrin Activation. Biophys J [Internet]. 2019;116(6):1000-10. Available from: https://doi.org/10.1016/j.bpj.2019.02.011

30. Kalli AC, Wegener KL, Goult BT, Anthis NJ, Campbell ID, Sansom MSP. The Structure of the Talin/Integrin Complex at a Lipid Bilayer: An NMR and MD Simulation Study. Structure [Internet]. 2010;18(10):1280-8. Available from: http://dx.doi.org/10.1016/j.str.2010.07.012

31. Li H, Deng Y, Sun K, Yang H, Liu J, Wang M, et al. Structural basis of kindlin-mediated integrin recognition and activation. Proc Natl Acad Sci. 2017;114(35):9349-54.

32. Weljie AM, Hwang PM, Vogel HJ, Misra S, Plow EF, Qin J. Solution structures of the cytoplasmic tail complex from platelet integrin alpha IIb- and beta 3-subunits. Proc Natl Acad Sci U S A [Internet]. 2002 Apr 30 [cited 2019 May 20];99(9):5878-83. Available from: http://www.ncbi.nlm.nih.gov/pubmed/11983888

33. Li H, Deng Y, Sun K, Yang H, Liu J, Wang M, et al. Structural basis of kindlin-mediated integrin recognition and activation. Proc Natl Acad Sci U S A [Internet]. 2017 Jul 29 [cited 2018 May 22];114(35):9349-54. Available from: http://www.ncbi.nlm.nih.gov/pubmed/28739949

34. Jorgensen WL, Chandrasekhar J, Madura JD, Impey RW, Klein ML. Comparison of simple potential functions for simulating liquid water. J Chem Phys [Internet]. 1983 Jul 15 [cited 2018 Mar 9];79(2):926-35. Available from:

http://aip.scitation.org/doi/10.1063/1.445869

35. Phillips JC, Braun R, Wang W, Gumbart J, Tajkhorshid E, Villa E, et al. Scalable molecular dynamics with NAMD. J Comput Chem [Internet]. 2005 Dec [cited 2014 Jul 9];26(16):1781-802. Available from:

http://www.pubmedcentral.nih.gov/articlerender.fcgi?artid=2486339\&tool=pmcentrez\&re ndertype $=$ abstract

36. Humphrey W, Dalke A, Schulten K. VMD: visual molecular dynamics. J Mol Graph [Internet]. 1996 Feb [cited 2018 Mar 9];14(1):33-8, 27-8. Available from: http://www.ncbi.nlm.nih.gov/pubmed/8744570

37. Bengtsson H, Corrada Bravo H, Gentleman R, Hossjer O, Jaffee H, Jiang D, et al. Package "matrixStats" Title Functions that Apply to Rows and Columns of Matrices (and to Vectors) [Internet]. 2018 [cited 2019 May 20]. Available from: https://cran.rproject.org/web/packages/matrixStats/matrixStats.pdf

38. Package "gplots" Title Various R Programming Tools for Plotting Data [Internet]. 2019 [cited 2019 May 20]. Available from: https://cran.rproject.org/web/packages/gplots/gplots.pdf

39. Grant BJ, Rodrigues APC, ElSawy KM, McCammon JA, Caves LSD. Bio3d: an R package for the comparative analysis of protein structures. Bioinformatics [Internet]. 2006 Nov 1 [cited 2018 Mar 9];22(21):2695-6. Available from: http://www.ncbi.nlm.nih.gov/pubmed/16940322

40. Costescu BI, Gräter F. Time-resolved force distribution analysis. BMC Biophys [Internet]. 
2013 Dec 1 [cited 2019 May 20];6(1):5. Available from: https://bmcbiophys.biomedcentral.com/articles/10.1186/2046-1682-6-5

41. Liu J, Wang Z, Thinn AMM, Ma Y-Q, Zhu J. The dual structural roles of the membrane distal region of the -integrin cytoplasmic tail during integrin inside-out activation. J Cell Sci. 2015;128(9):1718-31.

42. Guo J, Zhang Y, Li H, Chu H, Wang Q, Jiang S, et al. Intramembrane ionic protein-lipid interaction regulates integrin structure and function. PLoS Biol. 2018;16(11):1-28.

43. Eng ET, Smagghe BJ, Walz T, Springer TA. Intact alphaIIbbeta3 integrin is extended after activation as measured by solution X-ray scattering and electron microscopy. J Biol Chem [Internet]. 2011 Oct 7 [cited 2019 May 22];286(40):35218-26. Available from: http://www.ncbi.nlm.nih.gov/pubmed/21832081

44. Shams H, Mofrad MRK. $\alpha$-Actinin Induces a Kink in the Transmembrane Domain of $\beta 3$ Integrin and Impairs Activation via Talin. Biophys J [Internet]. 2017;113(4):948-56. Available from: http://dx.doi.org/10.1016/j.bpj.2017.06.064 